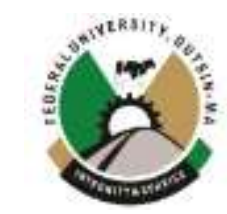

\title{
COMBINED EFFECTS OF VARIABLE VISCOSITY, VISCOUS DISSIPATION AND THERMAL RADIATION ON UNSTEADY NATURAL CONVECTION COUETTE FLOW THROUGH A VERTICAL POROUS CHANNEL
}

\author{
${ }^{* 1}$ Yusuf A. Bichi. and ${ }^{2}$ Ajibade, A.O. \\ ${ }^{1}$ Department of Mathematics, Federal University Dutsin-Ma, Katsina State, Nigeria. \\ ${ }^{2}$ Department of Mathematics, Ahmadu Bello University Zaria, Kaduna State, Nigeria. \\ *Corresponding author's E-mail: ayusuf@ fudutsinma.edu.ng
}

\begin{abstract}
The present article investigates combined effects of variable viscosity, viscous dissipation and thermal radiation on unsteady natural convection Couette flow through vertical porous channel. Non-linear Rosseland heat diffusion is deployed for the solution of the flow equations which as a consequence; together with the effects of variable viscosity, viscous dissipation and thermal radiation have resulted to high non-linear equations for the flow formation. Appropriate similarity variables are used to convert the partial differential equations associated with the fluid formation into dimensionless ordinary differential equations (ODEs) and the emerging ODEs are solved using Adomian decomposition method (ADM) and computer aided algebra package. Influences of the physical parameters involved in the problem are presented, discussed and conclusions are drawn. During the investigation; it was found that the fluid velocity and temperature were found to increase with increase in Eckert number, viscosity variation and thermal radiation.
\end{abstract}

Keywords: Natural convection; Thermal radiation; Variable viscosity; Viscous dissipation; Adomian decomposition method.

\section{INTRODUCTION}

Couette flow is a phenomenon in fluid flow which occurs due to the movement of bounding surface surrounding the fluid. This type of flow occurs in fluid machinery involving moving parts; especially in hydrodynamics lubrication where it has been used as a fundamental method for measurement of viscosity and also as a means of estimating drag force in many wall driven applications (Yasutomi (1984)).

Natural convection flow is a phenomenon in fluid flow where the fluid motion is induced by density difference occurring between the fluid molecules. In this mechanism, the fluid molecules surrounding the heat source receives heat, becomes less dense and raised, the surrounding cooler fluid then moves to replace it. Natural convection flows occur in many technological and industrial applications such as in nuclear reactors, rapid cooling process, motion of fluid in computer equipment, radiators, storage devices, cooling of electronic equipments inside computers, furnaces etc.

Study of fluid flows with variable viscosity through porous media has been investigated by researchers due to its numerous applications in sciences and engineering technology; particularly in the utilization of geothermal energy, high performance building insulation, crude oil extraction in petroleum industries, chemical catalytic reactor, underground disposal of nuclear waste materials and many others; refer to Ingham and Pop (1998) and Neild and Bejan (2013). The usual assumption of constant viscosity property of fluids evaluated at some reference temperature is not enough to depict a true situation in the flow characteristics in boundary layer flows. To accurately predict the flow and heat transfer rates; it is necessary to take into account temperature-dependent viscosity of fluid in boundary layer flows as increase in temperature leads to the increase in transport phenomena by reducing the fluid viscosity across the boundary layer. In related studies; Gray et al. (1982) and Mehta and Sood (1992) submitted that when varying viscosity property of fluid is included the flow characteristics change substantially compared to the constant case. Other correlated studies can be seen in Lai and Kulacki (1990), Sahin (1999), Hossain et al. (2001),Salem (2007), Seddeek and Salama (2007) and Yusuf and Ajibade (2018b).

Viscous dissipation is a phenomenon where by useful energy of a system is converted into its internal energy and as a result this amounts to poor performance of the system. Several scholars have investigated to this effect and can be seen in Soundalgekar (1972), Isreal-Cookey et al. (2003), Alam et al. (2006) and Salem (2013); few to report among others.

In engineering processes; when a system is at a state of work, some of its energy is emitted to the surrounding environment in the form of electromagnetic waves termed as "thermal radiation" and this result in poor performance of the system. For minimization of thermal radiation by systems, correlated studies were conducted to this effect and can be viewed in Ibanez et al. (2003), Makinde et al. (2007), Makinde (2008), Ajibade et al. (2011) and Makinde and Ogulu (2011) where the latter 
researchers concluded that an increase in the positive value of viscosity variation parameter resulted into a decrease in the fluid velocity. Some researchers in the aforementioned studies discussed the effect of thermal radiation using linearized Rosseland heat diffusion and this was however criticized by Magyari and Pantokratoras (2011); arguing that it does not mirrors a correct mechanism in energy transfer in most boundary layer flows and they therefore suggested alternative approach using non-linear Rosseland heat diffusion. In recognition of this achievement; correlated studies can be viewed in Yabo et al. (2016), Jha et al. (2017), Yusuf and Ajibade (2018a, 2019).

The present article extends the study of Yusuf and Ajibade (2018b) which neglected the effect of viscous dissipation while considering temperature-dependent viscosity in their flow formation. Here, our model incorporates the effect of viscous dissipation in order to capture more realistic behavior in the flow phenomenon. Similarly; the idea of Magyari and Pantokratoras (2011) for expanding radiative heat flux and that of Carey and Mollendorf (1978) for expressing dynamic fluid viscosity are assumed.

\section{MATHEMATICAL PROBLEM}

The physical problem under consideration consists of a vertical channel formed by two infinite parallel plates kept $\mathrm{h}$ distance apart with the channel filled with an optically thick incompressible viscous fluid in the presence of an incidence radiative heat flux of intensity $q_{r}$ which is absorbed by the plates and transferred to the fluid as shown in figure 1 below. The fluid properties are all assumed to be constant except for its viscosity which is temperature-dependent. Since the fluid is optically thick, the radiative heat flux in the flow formation is expanded using non-linear Rosseland heat diffusion. At time $t \leq 0$, both the fluid and the plates are assumed to be at rest with constant temperature $T_{0}$. At time $t>0$, the temperature of the plate kept at $y^{\prime}=0$ rise to $T_{w}$ while the other plate at $y^{\prime}=h$ is fixed and maintained at temperature $T_{0}$. The stream wise coordinate is denoted by $x^{\prime}$ - axis taken along the channel in the vertically upward direction and that normal to it is denoted by $y^{\prime}$. Fully developed flow is considered in this model meaning that the axial ( $x^{\prime}$-direction) velocity depends only on the transverse co-ordinate $y^{\prime}$. Furthermore; in the flow, the effect of viscous dissipation is taken into account while that of the radiative heat flux in the $x^{\prime}$ direction is assumed to be negligible compared to that in the $y^{\prime}$ - direction. Since the plates are of infinite length, the velocity and temperature are functions of $y^{\prime}$ and t only.

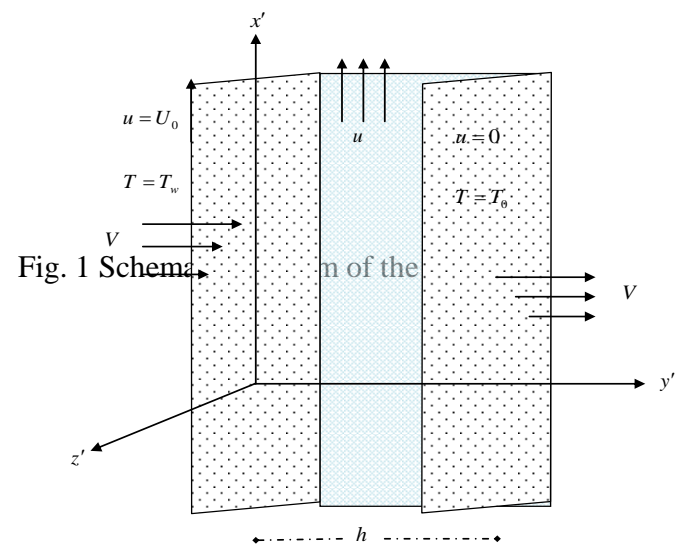

Under these assumptions; the appropriate governing equations in dimensional form are:

$$
\begin{aligned}
& \frac{\partial u}{\partial t}+V \frac{\partial u}{\partial y^{\prime}}=\frac{1}{\rho}\left(\mu \frac{\partial u}{\partial y^{\prime}}\right)+g \beta\left(T-T_{0}\right) \\
& \frac{\partial T}{\partial t}+V \frac{\partial T}{\partial y^{\prime}}=\alpha\left(\frac{\partial^{2} T}{\partial y^{\prime 2}}-\frac{1}{k} \frac{\partial q_{r}}{\partial y^{\prime}}\right)+\frac{1}{\rho c_{p}}\left(\frac{\partial u}{\partial y^{\prime}}\right)^{2}
\end{aligned}
$$

Following Carey and Mollendorf (1978), the fluid dynamic viscosity $(\mu)$ is expressed in the form:

$$
\mu=\mu_{0}\left(1-\lambda\left(\frac{T-T_{0}}{T_{w}-T_{0}}\right)\right) \quad \text { for } \quad \lambda \in \mathfrak{R}
$$


with the radiative heat flux $\left(q_{r}\right)$ following Sparrow and Cess (1978) as:

$$
q_{r}=\frac{-4 \sigma \partial T^{4}}{3 \delta \partial y^{\prime}}
$$

and the initial and boundary conditions for the velocity and temperature fields as:

$$
\begin{gathered}
u=U_{0}, \quad T=T_{w} \quad \text { at } \quad y^{\prime}=0 \\
u=0, \quad T=T_{w} \quad \text { at } \quad y^{\prime}=h
\end{gathered}
$$

\section{METHOD OF SOLUTION:}

In order to transform the governing equations (1) and (2) together with the boundary conditions (5) and (6) into dimensionless form; the following similarity variables (Makinde et al. (2007)) are used:

$$
\begin{aligned}
& u=U_{0}, \quad T=T_{w} \quad \text { at } \quad y^{\prime}=0 \\
& u=U_{0} f(y), \quad y=\frac{y^{\prime}}{\sqrt{v t}}, \quad \theta(y)=\frac{T-T_{0}}{T_{w}-T_{0}}, \quad \delta=2 \sqrt{v t}
\end{aligned}
$$

Using equations (3) and (7) in equation (1), the following momentum equation is obtained:

$$
\begin{aligned}
f^{\prime \prime}(y)=- & \frac{1}{2}(y+c) f^{\prime}(y)(1+\lambda \theta(y))+\lambda \theta^{\prime}(y) f^{\prime}(y)(1+\lambda \theta(y)) \\
& -G r \theta(y)(1+\lambda \theta(y)), \quad \lambda \in \mathfrak{R}
\end{aligned}
$$

Following Magyari and Pantokratoras (2011) and using equation (7); the term containing the radiative heat flux i.e. $\frac{\partial q_{r}}{\partial y^{\prime}}$ is expanded as follow:

$$
\begin{gathered}
\frac{\partial q_{r}}{\partial y^{\prime}}=\frac{\partial}{\partial y^{\prime}}\left[\left(-\frac{4 \sigma}{3 \delta}\right) \frac{\partial T^{4}}{\partial y^{\prime}}\right]=-\frac{4 \sigma}{3 v t 2 \sqrt{v t}(1-\lambda \theta(y))}\left(\frac{\partial T^{4}}{\partial y^{2}}\left(\left[\theta(y)\left(T_{w}-T_{0}\right)+T_{0}\right]^{4}\right)\right) \\
=-\frac{4 \sigma}{3 v t 2 \sqrt{v t}(1-\lambda \theta(y))}\left(\frac{\partial}{\partial y}\left(4\left[\theta(y)\left(T_{w}-T_{0}\right)+T_{0}\right]^{3}\right) \frac{\partial}{\partial y}\left(\theta(y)\left(T_{w}-T_{0}\right)\right)\right) \\
=-\frac{4 \sigma}{3 v t 2 \sqrt{v t}(1-\lambda \theta(y))}\left(12\left[\theta(y)\left(T_{w}-T_{0}\right)+T_{0}\right]^{2} \frac{\partial}{\partial y}\left(\theta(y)\left(T_{w}-T_{0}\right)\right) \frac{\partial}{\partial y}\left(\theta(y)\left(T_{w}-T_{0}\right)\right)\right) \\
\quad-\frac{4 \sigma}{3 v t 2 \sqrt{v t}(1-\lambda \theta(y))}\left(4\left[\theta(y)\left(T_{w}-T_{0}\right)+T_{\infty}\right]^{3} \frac{\partial^{2}}{\partial y^{2}}\left(\theta(y)\left(T_{w}-T_{0}\right)\right)\right)
\end{gathered}
$$

Substituting equation (7) and (9) into equation (2) and simplifying gives the following energy equation :

$$
\begin{aligned}
\theta^{\prime \prime}(y)= & -\frac{\operatorname{Pr}}{2}\left(1-\frac{1}{2} \lambda \theta(y)\right)\left(y\left(1-\frac{1}{2} \lambda \theta(y)\right)+c\right) \theta^{\prime}(y)\left[1-\frac{4 R}{3}[\theta(y)+\phi]^{3}\right] \\
& -4 R[\theta(y)+\phi]^{2} \theta^{\prime 2}(y)\left[1-\frac{4 R}{3}[\theta(y)+\phi]^{3}\right] \\
& -\operatorname{Pr} E c f^{\prime 2}(y)(1-\lambda \theta(y))\left[1-\frac{4 R}{3}[\theta(y)+\phi]^{3}\right]
\end{aligned}
$$

Using equation (7) in equation (5) and (6) is as follow:

$$
u=U_{0}, T=T_{w} \text {, at } y^{\prime}=0
$$




$$
\begin{aligned}
& \Rightarrow u=U_{0} f(0), \quad \theta(0)=\frac{T-T_{0}}{T_{w}-T_{0}}, \quad y \sqrt{v t}=0 \\
& \Rightarrow f(0)=\theta(0)=1 \quad \text { at } \quad y=0 \\
& \text { and } \quad u=0, \quad T=T_{0} \quad \text { at } \quad y^{\prime}=h \\
& \Rightarrow 0=U_{0} f(H), \quad \theta(H)=0 \quad \text { at } \quad y=H \\
& \Rightarrow f(H)=\theta(H)=0 \quad \text { at } \quad y=H \\
& \text { where } R=\frac{4 \sigma\left(T_{w}-T_{0}\right)^{3}}{3 k \delta}, \quad \phi=\frac{T_{0}}{T_{w}-T_{0}}, \quad E c=\frac{U_{0}^{2}}{c_{p}\left(T_{w}-T_{0}\right)} \text {, } \\
& G r=\frac{g \beta\left(T_{w}-T_{0}\right) \delta}{4 v U_{0}}, \quad c=\frac{-2 V \sqrt{t}}{\sqrt{v}}
\end{aligned}
$$

\section{Adomian decomposition solution of the problem:}

Equations (8) and (10) under the boundary conditions (11) and (12) are solved using ADM as follow:

Denote by $f^{\prime \prime}=\frac{d^{2} f}{d y^{2}}, \theta^{\prime \prime}=\frac{d^{2} \theta}{d y^{2}}$ and let $L(y)=\frac{d^{2} f}{d y^{2}}$

so that $L f(y)=f^{\prime \prime}(y), \quad L \theta(y)=\theta^{\prime \prime}(y)$ and $L^{-1}=\iint() d y d$.

Using equations (14); equation (9) and (10) can be written as:

$$
\begin{aligned}
L f(y)= & -\frac{1}{2}(y+c) f^{\prime}(y)(1+\lambda \theta(y))+\lambda \theta^{\prime}(y) f^{\prime}(y)(1+\lambda \theta(y))-G r \theta(y)(1+\lambda \theta(y)) \\
L \theta(y)= & -\frac{\operatorname{Pr}}{2}\left(1-\frac{1}{2} \lambda \theta(y)\right)\left(y\left(1-\frac{1}{2} \lambda \theta(y)\right)+c\right) \theta^{\prime}(y)\left[1-\frac{4 R}{3}[\theta(y)+\phi]^{3}\right] \\
& -4 R[\theta(y)+\phi]^{2} \theta^{\prime}(y) \theta^{\prime}(y)\left[1-\frac{4 R}{3}[\theta(y)+\phi]^{3}\right] \\
& -\operatorname{Pr}(1-\lambda \theta(y)) E c f^{\prime 2}(y)\left[1-\frac{4 R}{3}[\theta(y)+\phi]^{3}\right]
\end{aligned}
$$

Operating $L^{-1}$ to both sides of equation (15) and (16) we achieved:

$$
\begin{aligned}
L^{-1} L f(y)= & -\frac{1}{2} L^{-1}\left((y+c) f^{\prime}(y)(1+\lambda \theta(y))\right)+\lambda L^{-1}\left(\theta^{\prime}(y) f^{\prime}(y)(1+\lambda \theta(y))\right) \\
& -G r L^{-1}(\theta(y)(1+\lambda \theta(y))) \\
L^{-1} L \theta(y)= & -\frac{\operatorname{Pr}}{2} L^{-1}\left(\left(1-\frac{1}{2} \lambda \theta(y)\right)\left(y\left(1-\frac{1}{2} \lambda \theta(y)\right)+c\right) \theta^{\prime}(y)\left[1-\frac{4 R}{3}[\theta(y)+\phi]^{3}\right]\right) \\
& -4 R L^{-1}\left([\theta(y)+\phi]^{2} \theta^{\prime}(y) \theta^{\prime}(y)\left[1-\frac{4 R}{3}[\theta(y)+\phi]^{3}\right]\right) \\
- & \operatorname{Pr} L^{-1}\left((1-\lambda \theta(y)) E c f^{\prime 2}(y)\left[1-\frac{4 R}{3}[\theta(y)+\phi]^{3}\right]\right)
\end{aligned}
$$


But by ADM: $\quad L^{-1} L f(y)=f(y)-f(0)-y f^{\prime}(0)$

$$
L^{-1} L \theta(y)=\theta(y)-\theta(0)-y \theta^{\prime}(0)
$$

Using equations (11), (19) and (20) in equations (17) and (18) we have:

$$
\begin{aligned}
f(y)=1+y A- & \frac{1}{2} L^{-1}\left((y+c) f^{\prime}(y)(1+\lambda \theta(y))\right)+\lambda L^{-1}\left(\theta^{\prime}(y) f^{\prime}(y)(1+\lambda \theta(y))\right) \\
& -G r L^{-1}(\theta(y)(1+\lambda \theta(y))) \text { and } \\
\theta(y)= & 1+y B-\frac{\operatorname{Pr}}{2} L^{-1}\left(\left(1-\frac{1}{2} \lambda \theta(y)\right)\left(y\left(1-\frac{1}{2} \lambda \theta(y)\right)+c\right) \theta^{\prime}(y)\left[1-\frac{4 R}{3}[\theta(y)+\phi]^{3}\right]\right) \\
& -4 R L^{-1}\left([\theta(y)+\phi]^{2} \theta^{\prime}(y) \theta^{\prime}(y)\left[1-\frac{4 R}{3}[\theta(y)+\phi]^{3}\right]\right) \\
& -\operatorname{Pr} L^{-1}\left((1-\lambda \theta(y)) E c f^{\prime 2}(y)\left[1-\frac{4 R}{3}[\theta(y)+\phi]^{3}\right]\right)
\end{aligned}
$$

where $A, B$ are to be determined based on equation (12).

According to the standard ADM, $f(y)$ and $\theta(y)$ can be expressed as:

$$
f(y)=\sum_{n=0}^{\infty} f_{n}(y), \theta(y)=\sum_{n=0}^{\infty} \theta_{n}(y)
$$

Substituting equations (23) in equations (21) and (22), the following equations are realized:

$$
\begin{aligned}
& \sum_{n=0}^{\infty} f_{n}(y)=1+y A-\frac{1}{2} L^{-1}\left((y+c) \frac{d}{d y}\left(\sum_{n=0}^{\infty} f_{n}(y)\right)\left(1+\lambda \sum_{n=0}^{\infty} \theta_{n}(y)\right)\right) \\
& +\lambda L^{-1}\left(\frac{d}{d y}\left(\sum_{n=0}^{\infty} \theta_{n}(y)\right) \frac{d}{d y}\left(\sum_{n=0}^{\infty} f_{n}(y)\right)\left(1+\lambda \sum_{n=0}^{\infty} \theta_{n}(y)\right)\right) \\
& -G r L^{-1}\left(\sum_{n=0}^{\infty} \theta_{n}(y)\left(1+\lambda \sum_{n=0}^{\infty} \theta_{n}(y)\right) \quad\right. \text { and } \\
& \sum_{n=0}^{\infty} \theta_{n}(y)=1+y B-\frac{\operatorname{Pr}}{2} L^{-1}\left(\left(1-\frac{1}{2} \lambda \sum_{n=0}^{\infty} \theta_{n}(y)\right)\left(y\left(1-\frac{1}{2} \lambda \sum_{n=0}^{\infty} \theta_{n}(y)\right)+c\right) \frac{d}{d y}\left(\sum_{n=0}^{\infty} \theta_{n}(y)\right)\left[1-\frac{4 R}{3}\left[\sum_{n=0}^{\infty} \theta_{n}(y)+\phi\right]^{3}\right]\right) \\
& -4 R L^{-1}\left(\left[\sum_{n=0}^{\infty} \theta_{n}(y)+\phi\right]^{2} \frac{d}{d y}\left(\sum_{n=0}^{\infty} \theta_{n}(y)\right) \frac{d}{d y}\left(\sum_{n=0}^{\infty} \theta_{n}(y)\right)\left[1-\frac{4 R}{3}\left[\sum_{n=0}^{\infty} \theta_{n}(y)+\phi\right]^{3}\right]\right) \\
& -\operatorname{Pr} L^{-1}\left(\left(1-\lambda \sum_{n=0}^{\infty} \theta_{n}(y)\right) E c \frac{d}{d y}\left(\sum_{n=0}^{\infty} f_{n}(y)\right) \frac{d}{d y}\left(\sum_{n=0}^{\infty} f_{n}(y)\right)\left[1-\frac{4 R}{3}\left[\sum_{n=0}^{\infty} \theta_{n}(y)+\phi\right]^{3}\right]\right) \\
& f_{j+1}(y)=-\frac{1}{2} L^{-1}\left((y+c) \frac{d}{d y}\left(f_{j}(y)\right)\left(1+\lambda \theta_{j}(y)\right)\right)+\lambda L^{-1}\left(\frac{d}{d y}\left(\theta_{j}(y)\right) \frac{d}{d y}\left(f_{j}(y)\right)\left(1+\lambda \theta_{j}(y)\right)\right)
\end{aligned}
$$




$$
\begin{aligned}
\theta_{j+1}(y) & =1+y B-\frac{\operatorname{Pr}}{2} L^{-1}\left(\left(1-\frac{1}{2} \lambda \theta_{j}(y)\right)\left(y\left(1-\frac{1}{2} \lambda \theta_{j}(y)\right)+c\right) \frac{d}{d y}\left(\theta_{j}(y)\right)\left[1-\frac{4 R}{3}\left[\theta_{j}(y)+\phi\right]^{3}\right]\right) \\
& -4 R L^{-1}\left(\left[\theta_{j}(y)+\phi\right]^{2} \frac{d}{d y}\left(\theta_{j}(y)\right) \frac{d}{d y}\left(\theta_{j}(y)\right)\left[1-\frac{4 R}{3}\left[\theta_{j}(y)+\phi\right]^{3}\right]\right) \\
& -\operatorname{Pr} L^{-1}\left(\left(1-\lambda \theta_{j}(y)\right) E c \frac{d}{d y}\left(f_{j}(y)\right) \frac{d}{d y}\left(f_{j}(y)\right)\left[1-\frac{4 R}{3}\left[\theta_{j}(y)+\phi\right]^{3}\right]\right)
\end{aligned}
$$

Finally; the solution is given by the partial sum:

$$
f(y)=\sum_{j=0}^{S} f_{j} \quad \text { and } \quad \theta(y)=\sum_{j=0}^{Q} \theta_{j}
$$

where $S$ and $Q$ are truncation points for which the ADM solution converges.

For details on ADM; refer to Adomian (1994).

Convergence of the ADM solution and termination criterion of the problem:

Convergence of Adomian decomposition solution has been assured to be rapidly in Adomian (1994) and Charrault (1990). Nevertheless; to verify the convergence of the ADM solution in the present problem; the method of ratio test is deployed. Using computer algebra package the following terms were obtained at

$$
\begin{aligned}
& y=0.5, \quad E c=0.01, \quad c=0.1, \quad \lambda=0.1, \quad G r=10, \quad \phi=0.1, \quad R=0.1, \quad \operatorname{Pr}=0.71 \quad \text { as: } \\
& \theta_{0}=0.520991 \theta_{1}=0.0264688, \theta_{2}=0.000596, \theta_{3}=0.000010 \\
& f_{0}=1.030495, f_{1}=-0.002539, f_{2}=0.001165, f_{3}=-0.000055
\end{aligned}
$$

the following ratios are evaluated as:

$$
\begin{aligned}
& \left|\frac{\theta_{1}}{\theta_{0}}\right|=0.958018,\left|\frac{\theta_{2}}{\theta_{1}}\right|=0.022517,\left|\frac{\theta_{3}}{\theta_{2}}\right|=0.016779 \\
& \left|\frac{f_{1}}{f_{0}}\right|=0.01447,\left|\frac{f_{2}}{f_{1}}\right|=0.078221,\left|\frac{f_{3}}{f_{2}}\right|=0.047082
\end{aligned}
$$

Numerical values attained in equation (31) are seen to agree with the ratio test formula for convergence since $\lim _{j \rightarrow \infty}\left|\frac{f_{j+1}}{f_{j}}\right|<1$,

for $\mathrm{j} \geq 0$ (Robert [32])

Hence the ADM solution of the present problem converges. For a meaningful solution, the series is truncated at a point such that the contribution of any additional term is negligible to the final solution. For this reason; a termination criterion is used such that the series is paused whenever $\left|u_{i}, \theta_{i}\right|<\varepsilon$ where we have chosen $\varepsilon=5.0 \times 10^{-5}$. Considering this assumption, the solution for $u$ and $\theta$ are thus truncated after the fourth terms. Due to huge size of the computed ADM solution, the final solution is not displayed here but is used for numerical computations for the purpose of discussing the result.

Nusselt number and skin friction on the channel plates:

Since the fluid viscosity considered in this extension has variable status, the skin frictions on the plates is evaluated following Kay (2017) using:

$$
\tau_{0}=\left.(1-\lambda \theta) \frac{d f}{d y^{\prime}}\right|_{y^{\prime}=0} \quad \text { and } \quad \tau_{1}=\left.(1-\lambda \theta) \frac{d f}{d y^{\prime}}\right|_{y^{\prime}=1}
$$

while the Nusselt numbers are is calculated via: 


$$
N u_{0}=-\left.\frac{d \theta}{d y^{\prime}}\right|_{y^{\prime}=0} \quad \text { and } \quad N u_{1}=-\left.\frac{d \theta}{d y^{\prime}}\right|_{y^{\prime}=1}
$$

\section{RESULTS AND DISCUSSION:}

Using computer algebra software package (Mathematica) the truncated solution of equation (29) is simulated and the results are presented on Figures 2- 12 and Tables I - III. For the purpose of discussion, the ambient Prandtl numbers are taken as 0.16, 0.44, 0.71 and 4 which correspond to mixture of noble gases, electrolyte solution, air and E-12 refrigerant respectively. Similarly, other values of Prandtl numbers, Eckert number, temperature difference parameter, suction parameter and viscosity variation parameter are chosen arbitrarily from 0 to 3 while that of Grashof numbers are taken as 10,12, 14 which correspond to the cooling of the plates by free convection. Furthermore; the values of thermal radiation (R) are carefully chosen in the range $0 \leq R \leq 0.5$ for which the ADM solution of equation (29) converges.

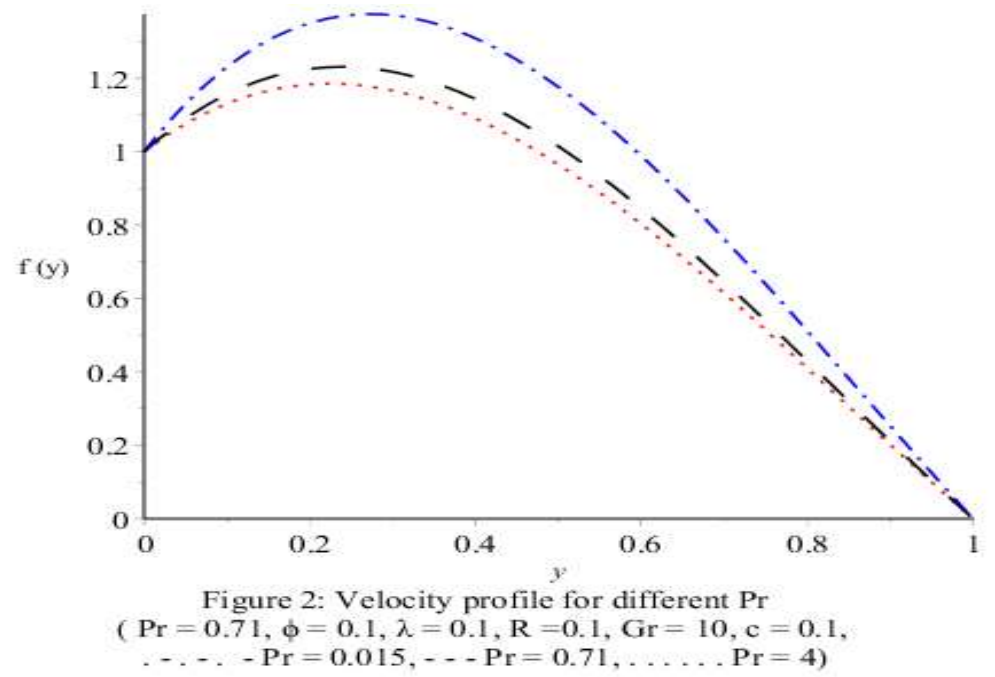

The effect of different values of $\operatorname{Pr}$ on the fluid velocity is depicted in Figure 2 above. This figure illustrates that the fluid velocity in the channel decreases with increase in Pr. This behavior is as a result of the decrease in thermal diffusion of the fluid.

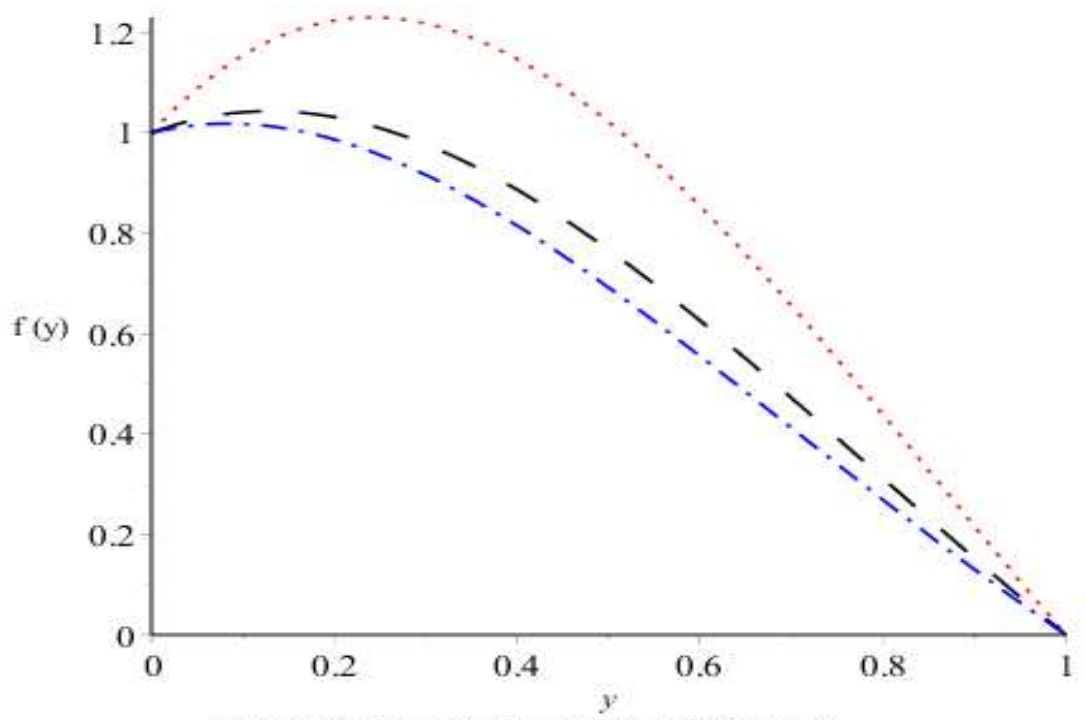

Figure 3: Velocity profile for different $\mathrm{R}$

$(\operatorname{Pr}=0.71, \lambda=0.1, \phi=0.1, \mathrm{c}=0.1, \mathrm{Ec}=0.1, \lambda=0.1, \ldots \mathrm{R}=0.1$,

$$
\cdots \mathrm{R}=0.5, \ldots . \mathrm{R}=1 \text { ) }
$$


Figure 3 reveals the effect of changing thermal radiation on the fluid velocity where it is exposed that the velocity decreases with increase in thermal radiation. This culture is the consequence of the decrease in thermal diffusion of the working fluid.

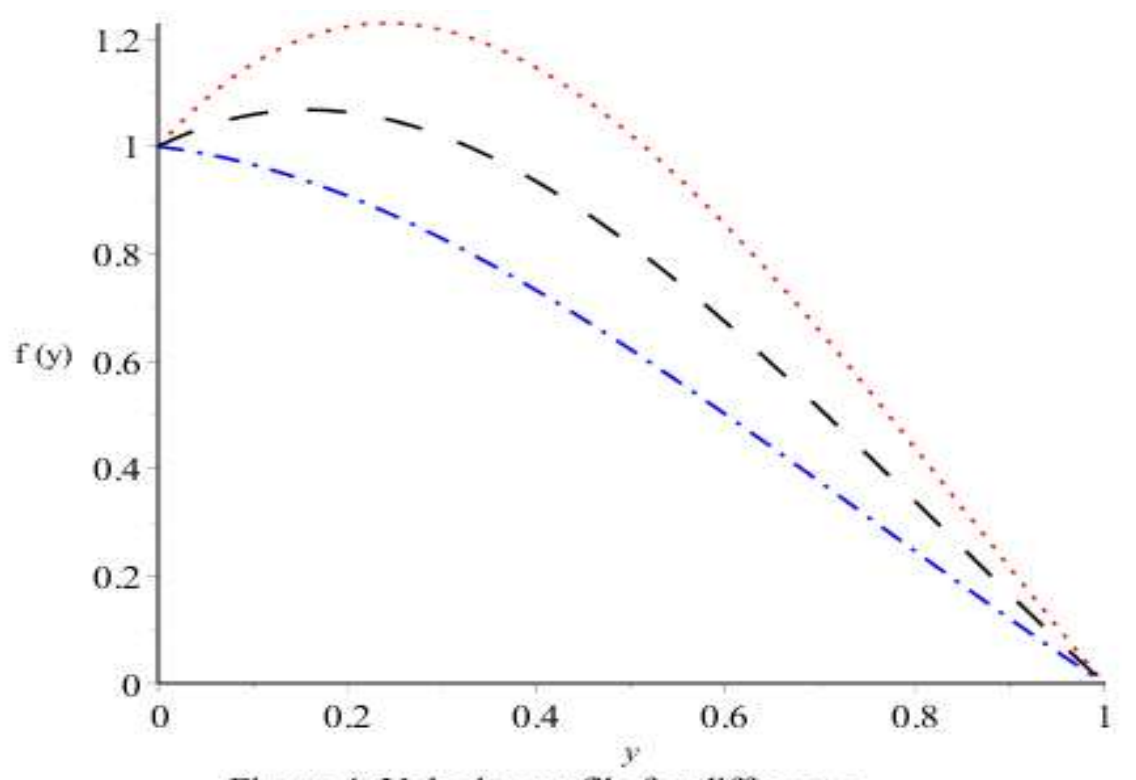

Figure 4: Velocity profile for different c

$$
\begin{gathered}
(\operatorname{Pr}=0.71, \lambda=0.1, \phi=0.1, \mathrm{R}=0.1, \mathrm{Gr}=10, \mathrm{c}=0.1, \lambda=0.1, \\
\ldots \mathrm{c}=0.1,-\mathrm{c}=0.5, \ldots . .-\mathrm{c}=1)
\end{gathered}
$$

Figure 4 demonstrates that an increase in fluid suction results to the decrease in the fluid velocity within the channel. This trend is due to the decrease in kinematic viscosity of the fluid.

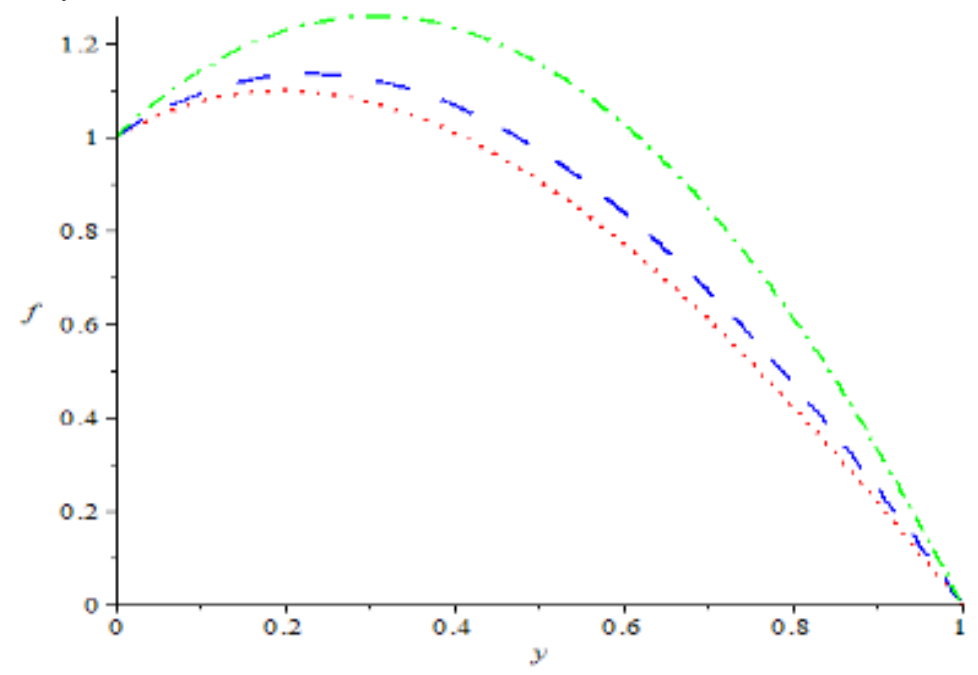

Fig. 5: velocity profiles for different values of Ec

$(\operatorname{Pr}=0.71, \mathrm{R}=0.1, \phi=0.1, \lambda=0.1, \mathrm{Gr}=5.0, \mathrm{c}=0.1, \ldots \ldots \mathrm{Ec}=0.1,-\cdots--\mathrm{Ec}=0.5, .-.-. . . \mathrm{Ec}=0.9)$

The effect of fluctuating Ec on the fluid velocity is pictured in figure 5. The figure shows that with increase in Ec; the velocity also increases with increase in Ec. This response is the consequential effect of the decrease in $\left(T_{w}-T_{0}\right)$. 


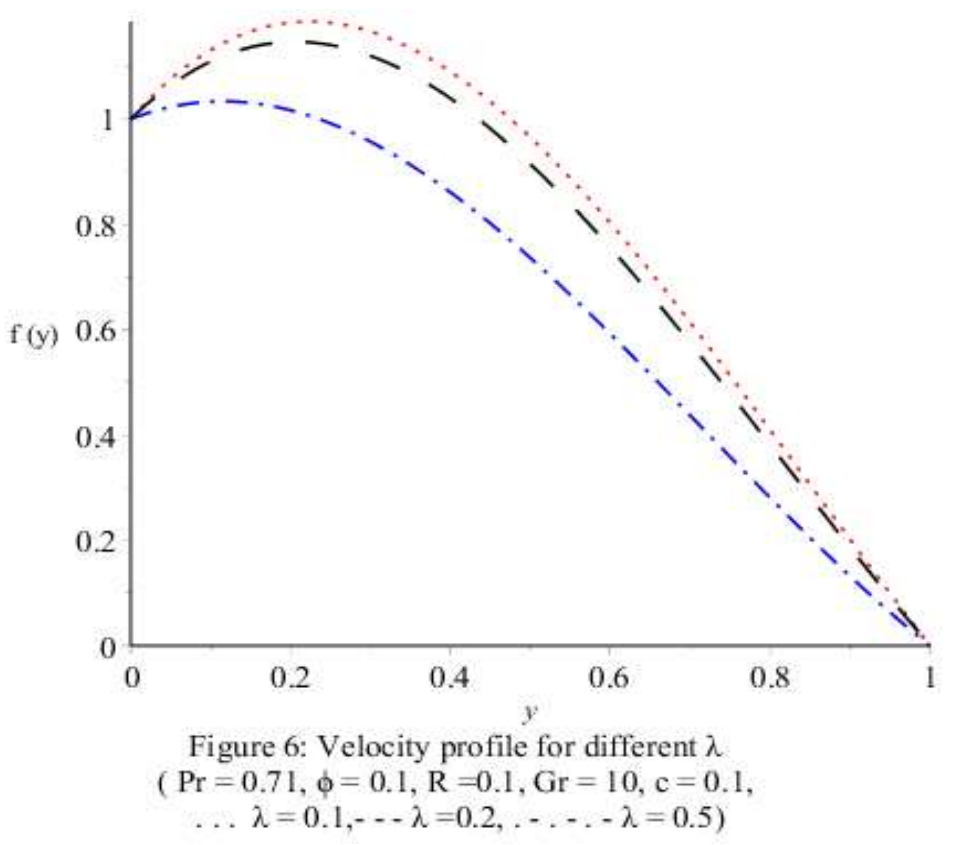

Figure 6 depicts the response of varying fluid viscosity on the velocity and the figure shows that the fluid velocity increases with decrease in viscosity (increasing value of $\lambda$ ).

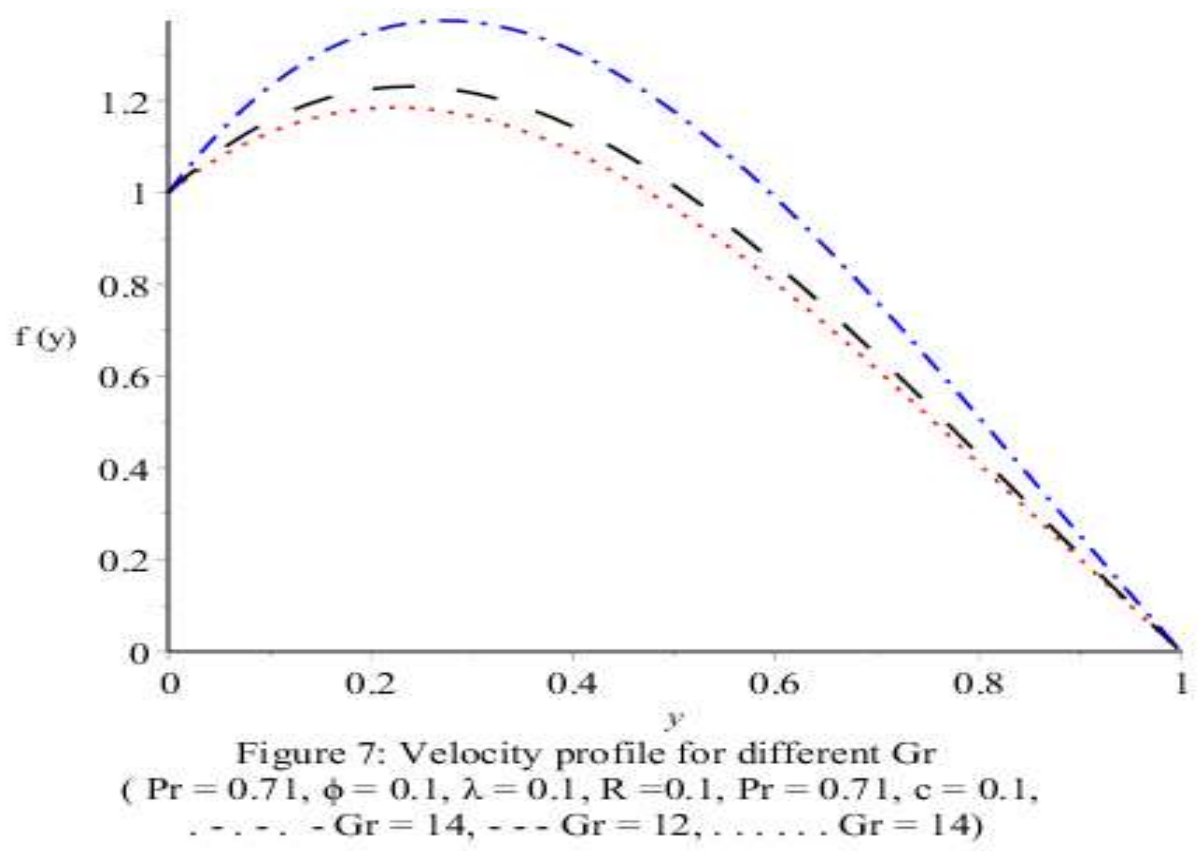

The effect of rising value of $\mathrm{Gr}$ is naked in figure 7 where the fluid velocity is observed to increase with growing Gr. This fashion is as result of the increase in buoyancy force of the fluid. 


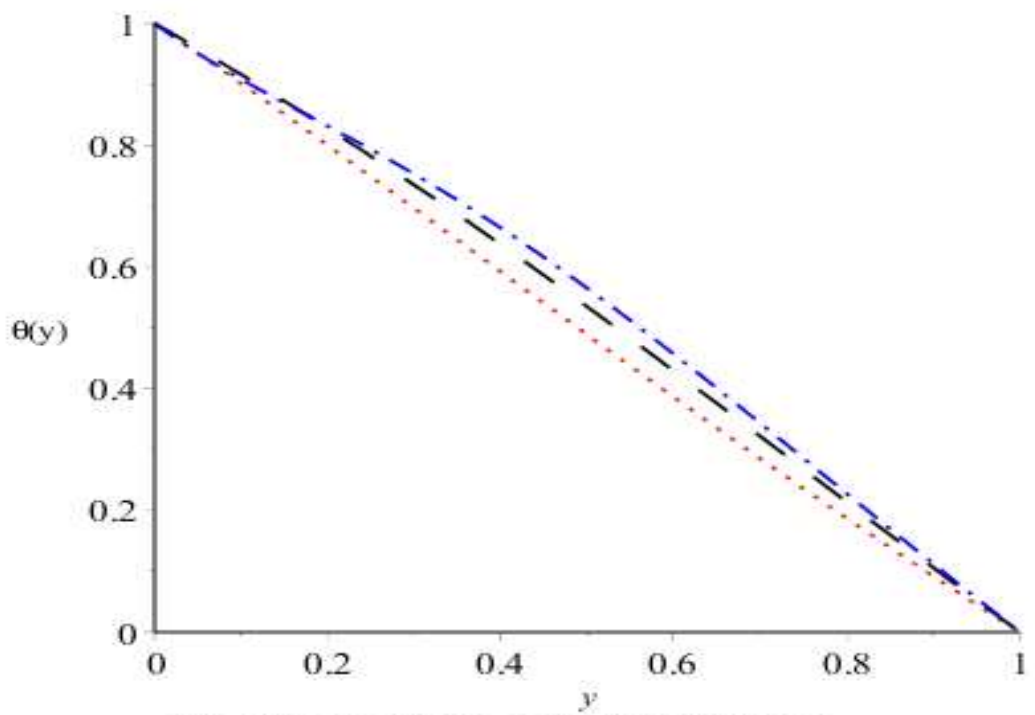

Fig 8: Temperature profile for different $\mathrm{R}$

$\operatorname{Pr}=0.71, \phi=0.1, \lambda=0.1, \mathrm{c}=0.1, \mathrm{Ec}=0.1, \ldots . \mathrm{R}=0.1, \ldots-\mathrm{R}=0.5,$. -...... $\mathrm{R}=1$

The response of different values of $\mathrm{R}$ is reflected in Figure 8. From this figure it is viewed that with increase in $\mathrm{R}$ the fluid temperature also increases. This behavior is the resultant effect of the decrease in thermal diffusion of the working fluid.

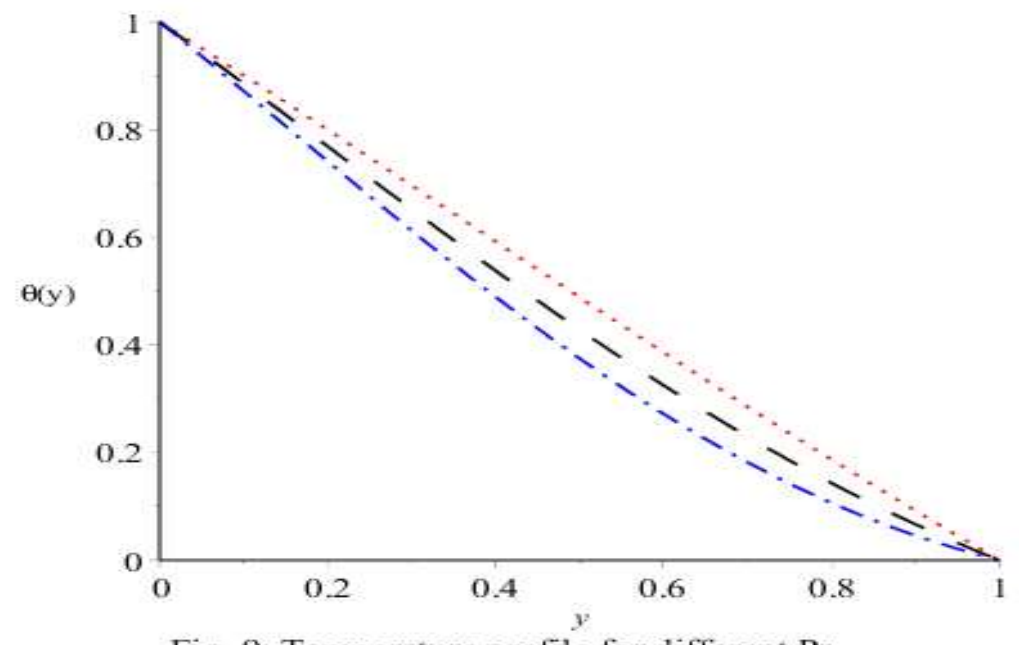

Fig. 9: Temperature profile for different $\mathrm{Pr}$

$\lambda=0.1, \phi=0.1, \mathrm{R}=0.1, \mathrm{c}=0.1, \mathrm{Ec}=0.1, \ldots . . \mathrm{Pr}=0.015, \ldots \mathrm{Pr}=$
$0.71, \ldots . . . \mathrm{Pr}=4$

Figure 9 shows the effect of different Pr on the fluid temperature within the channel where the temperature is seen to decrease with increase in Pr. This behavior is due to the decrease in thermal diffusion of the working fluid. 


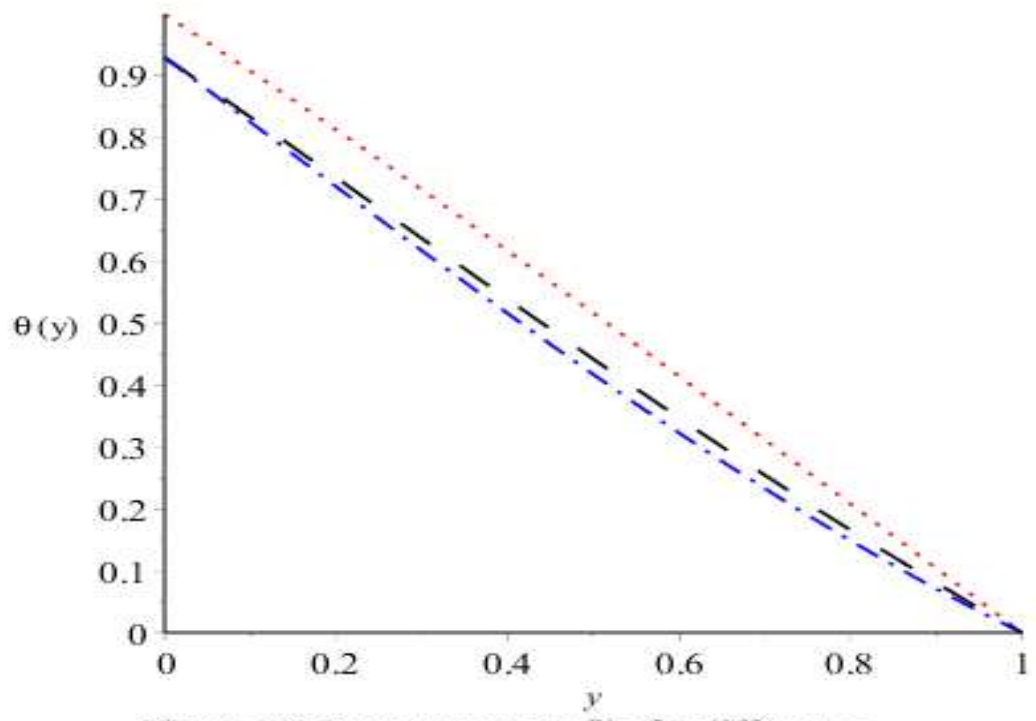

Figure 10: Temperature profile for different $\mathrm{c}$ $(\operatorname{Pr}=0.71, \lambda=0.1, \phi=0.1, \mathrm{R}=0.1, \mathrm{Ec}=0.1, \ldots \mathrm{c}=0.1$,$$
\ldots \mathrm{c}=0.5, \ldots .-\mathrm{c}=1 \text { ) }
$$

Figure 10 illustrates the effects of fluid suction on the fluid temperature in the channel. The figure shows that fluid temperature decreases with increase in suction. This development is as a result of the decrease in the kinematic viscosity of the fluid.

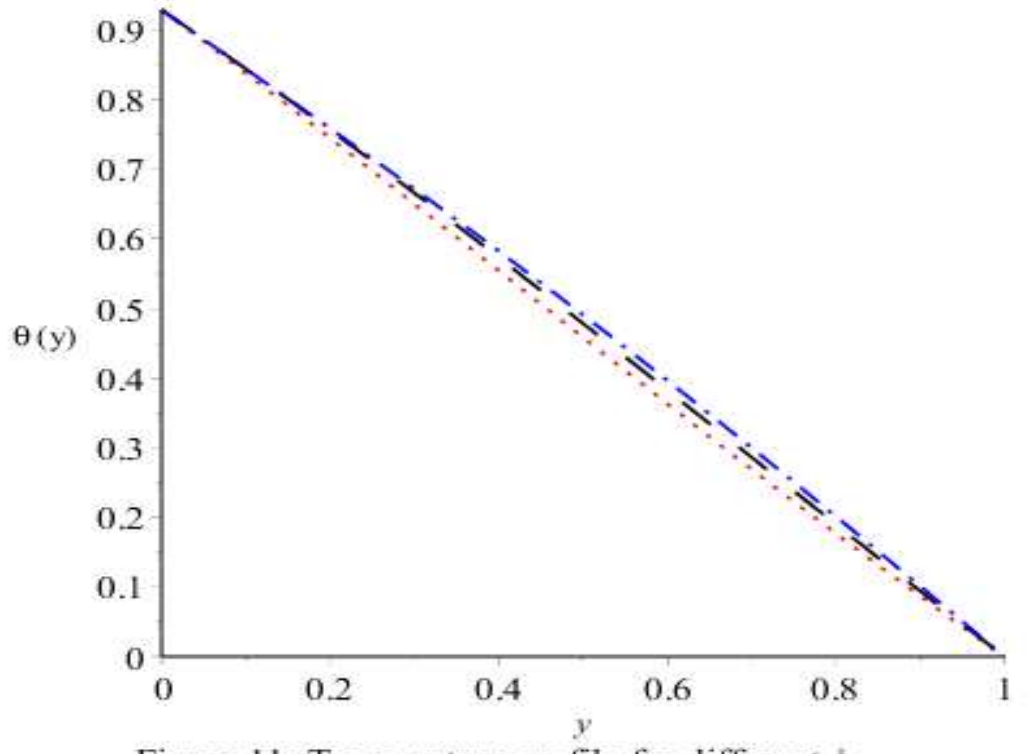

Figure 11: Temperature profile for different $\phi$ $(\operatorname{Pr}=0.71, \lambda=0.1, \mathrm{Ec}=0.1, \mathrm{R}=0.1, \mathrm{c}=0.1, \ldots \phi=0.1$, $\cdots \phi=0.5, \ldots . \phi=1$ )

The effect of fluctuating temperature difference $(\phi)$ is displayed in figure 11 where the fluid temperature is observed to increase with increase in $\phi$. This culture is inclined to the increase in the ambient temperature of the fluid. 


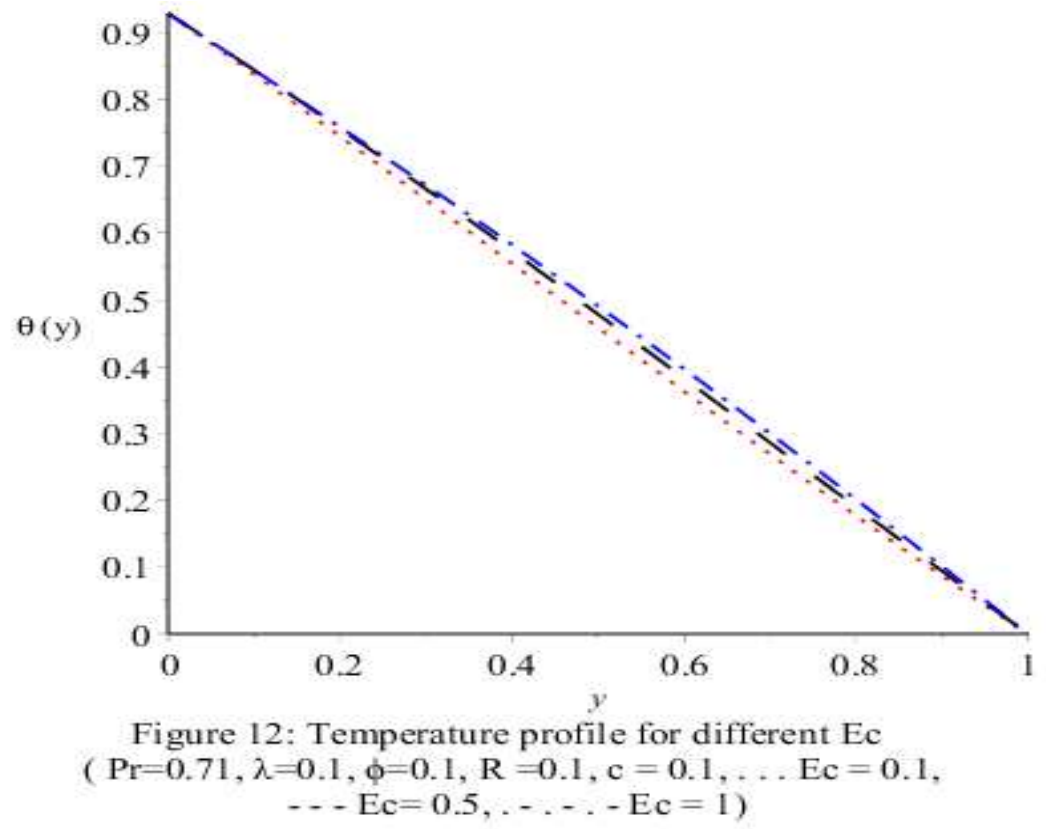

Effect of Ec on the fluid temperature is exposed in figure 12 where the fluid temperature is seen to increase with increase in Ec. This fashion is inclined to the decrease in temperature difference between the ambient and wall temperature. 
Table I: Skin frictions on the channel plates

\begin{tabular}{|l|l|l|l|l|l|c|}
\hline \multirow{2}{*}{$\lambda$} & \multicolumn{2}{|l|}{$\begin{array}{l}\mathrm{R}=0.1, \phi=0.21, \mathrm{Pr}=0.71, \\
\mathrm{Gr}=10, \mathrm{c}=0.001, \mathrm{Ec}=0.001\end{array}$} & \multicolumn{2}{l}{$\begin{array}{l}\mathrm{R}=0.2, \phi=0.21, \mathrm{Pr}=0.71, \\
\mathrm{Gr}=10, \mathrm{c}=0.001, \mathrm{Ec}=0.001\end{array}$} & \multicolumn{2}{l}{$\begin{array}{l}\mathrm{R}=0.1, \phi=0.5, \mathrm{Pr}=0.71, \\
\mathrm{Gr}=10, \mathrm{c}=0.001, \mathrm{Ec}=0.001\end{array}$} \\
\cline { 2 - 7 } & $\tau_{0}$ & $\tau_{1}$ & $\tau_{0}$ & $\tau_{1}$ & $\tau_{0}$ & 1.55302 \\
\hline 0.1 & 1.76195 & 1.39429 & 2.10482 & 2.03810 & 1.84734 & 2.95671 \\
\hline 0.3 & 1.12987 & 1.75274 & 1.95056 & 3.41679 & 1.66813 & 13.3163 \\
\hline 0.5 & 0.90462 & 108.491 & 0.91455 & 9.25520 & 1.61992 & 109.023 \\
\hline 0.7 & 0.59220 & 1021.6 & 0.811272 & 70.1430 & 0.808395 & \\
\hline
\end{tabular}

Table I shows the effects of varying parameters on the skin friction between the working fluid and the channel plates. For some fixed parameters and with rising value of $\lambda$; the skin friction o the heated plate $\left(\tau_{0}\right)$ is viewed to increase while it decreases on the cold plate $\left(\tau_{1}\right)$ with decrease in the fluid viscosity. Similarly; with small increase in thermal radiation (R), $\tau_{0}$ is observed to increase while $\tau_{1}$ is viewed to decreases and later the ski friction on the cold plate increases with more increase in $\lambda$. Furthermore; with minor increase in $\phi$, both $\tau_{0}$ and $\tau_{1}$ decreases and later $\tau_{1}$ increases with further increase in $\lambda$.

Table II: Nusselt number on the channel plates

\begin{tabular}{|c|c|c|c|c|c|c|}
\hline \multirow{2}{*}{$\operatorname{Pr}$} & \multicolumn{2}{|c|}{$\begin{array}{l}\mathrm{R}=0.1, \phi=0.21, \mathrm{Pr}=0.71 \\
\mathrm{Gr}=10, \mathrm{c}=0.001, \mathrm{Ec}=0.001\end{array}$} & \multicolumn{2}{|c|}{$\begin{array}{l}\mathrm{R}=0.3, \phi=0.21, \mathrm{Pr}=0.71 \\
\mathrm{Gr}=10, \mathrm{c}=0.001, \mathrm{Ec}=0.001\end{array}$} & \multicolumn{2}{|c|}{$\begin{array}{l}\mathrm{R}=0.3, \phi=0.5, \operatorname{Pr}=0.71 \\
\mathrm{Gr}=10, \mathrm{c}=0.001, \mathrm{Ec}=0.001\end{array}$} \\
\hline & $N u_{0}$ & $N u_{1}$ & $N u_{0}$ & $N u_{1}$ & $N u_{0}$ & $N u_{1}$ \\
\hline 0.016 & 0.883228 & 1.06150 & 0.80407 & 1.44585 & 0.90969 & 1.22324 \\
\hline 0.44 & 0.916847 & 1.04006 & 0.808645 & 1.14450 & 1.14147 & 1.19370 \\
\hline 0.71 & 0.954122 & 1.01027 & 0.96361 & 1.12088 & 1.34863 & 1.19572 \\
\hline 1 & 3.49130 & 89.0980 & 3.45396 & 56.1545 & 5.10622 & 90.5081 \\
\hline 2 & 2.13701 & 885.438 & 3.70684 & 577.975 & 7.16244 & 764.202 \\
\hline
\end{tabular}

Numerical values of Nusselt numbers on the plates are tabulated and displayed on Table II. It is viewed from the table that with fixed parameters and with increase in $\operatorname{Pr} N u_{0}$ is seen to increase while $N u_{1}$ is noticed to decrease. Similarly, with increase in R; $N u_{0}$ is watched to decreases while $N u_{1}$ is grasped to increase with ascending Pr and later both decreases with more increase in Pr. Furthermore; with slight increase in $\phi$ increases, $N u_{0}$ is grasped to increase while $N u_{1}$ decreases and later both $N u_{0}$ and $N u_{1}$ are viewed to increase with further increase in Pr. 


\section{CONCLUSION}

Combined effects of variable viscosity, viscous dissipation and thermal radiation on unsteady natural convection Couette flow through a vertical porous channel has been investigated using non-linear Rosseland heat diffusion and Adomian decomposition method. Using computer algebra package (Maple), equation (29) was coded and simulated with results presented on graphs and tables and were discussed. The major deductions from this investigation are:

i. Velocity of the fluid was established to increase with in decrease in viscosity of the fluid.

ii. Both the fluid's temperature and velocity were istituted to increase with increase in thermal radiation.

iii. Increase in Eckert number was found to increase the temperature and velocity of the fluid within the channel.

\section{ACKNOWLEDGEMENTS}

The researchers acknowledged the helping hand of Prof. Basant Kumar Jha of the Department of Mathematics, Ahmadu Bello University, Zaria, Kaduna State for tutoring, suggestive criticism and guidance. Similarly; the authors acknowledged Dr. Olumide Adesanya of the Department of Mathematics, Redeemers University, Ede, Osun State for tutoring, most especially on the concept of ADM.

\begin{tabular}{|c|c|c|}
\hline \multicolumn{3}{|c|}{ Nomenclature and Greek symbols: } \\
\hline Symbols & Interpretation & Unit \\
\hline$y^{\prime}$ & dimensional length & $m$ \\
\hline$y$ & dimensionless length & \\
\hline$t$ & time & $s$ \\
\hline $\mathrm{g}$ & gravitational acceleration & $m s^{-2}$ \\
\hline $\mathrm{k}$ & thermal conductivity & $W / m K$ \\
\hline$\delta$ & absorption coefficient & \\
\hline $\mathrm{T}$ & dimensional temperature of the fluid & $K$ \\
\hline$T_{w}$ & wall temperature & $K$ \\
\hline$T_{\infty}$ & ambient fluid temperature & K \\
\hline$V_{0}$ & velocity of suction & $m s^{-1}$ \\
\hline$u$ & dimensional velocity & \\
\hline$v$ & kinematic viscosity of the fluid & $m^{2} s^{-1}$ \\
\hline$\alpha$ & thermal diffusivity of the fluid & \\
\hline$\beta$ & volumetric expansion coefficient & $K^{-1}$ \\
\hline$\sigma$ & Stefan Boltzman constatnt & $J K^{-1}$ \\
\hline$\mu$ & variable viscosity & $\mathrm{kgm}^{-1} \mathrm{~s}^{-1}$ \\
\hline$q_{r}$ & radiative heat flux & $W m^{-2}$ \\
\hline
\end{tabular}

$\begin{array}{lll}\theta & \text { dimensionless temperature } & \\ U_{0} & \text { reference velocity } & \mathrm{ms}^{-1} \\ \lambda & \text { viscosity variation parameter } & \mathrm{K}^{-1} \\ \phi & \text { temperature difference parameter } & \mathrm{K} \\ R & \text { radiation parameter } & \\ \mathfrak{R} & \text { set of real numbers } & \\ \mathrm{Gr} & \text { Grashof number } & \\ \mathrm{Pr} & \text { Prandtl number } & \mathrm{kgm}^{-1} \mathrm{~s}^{-1} \\ \mu_{0} & \text { ambient fluid viscosity } & \\ \mathrm{c} & \text { suction parameter } & \\ \mathrm{Ec} & \text { Eckert number } & \\ N u_{0} & \text { Nusselt number on the heated plate } & \\ N u_{1} & \text { Nusselt number on the cold plate } & \\ \tau_{0} & \text { Skin friction on the heated plate } \\ \tau_{1} & \text { Skin friction on the cold plate } \\ \tau & \text { skin friction }\end{array}$

\section{REFERENCES}

Adomian, G. (1994). Solving Frontier Problems of Physics: The Decomposition Method. Boston, MA: Kluwer.

Ajibade, A. O., Jha B.K. and Andrew, O. (2011). Entropy Generation under the Effect of Suction/Injection. Journal of Applied Mathematical Modeling. 35: 4630-4646.

Alam M.M., Alim, M.A and Chaudhury (2006). Effect of viscous dissipation and pressure work in natural convection flow along vertical flat plate with heat conduction due to plate thickness. Journal of naval architecture and marine engineering 3(2006). pp. 69-76.

Carey,V.P. and Mollendorf, J. C. (1978). Natural convection in liquid with temperature dependent viscosity. In proceedings of the $6^{\text {th }}$ International C onference in Heat Transfer. Toronto. 2:211-217.

Cherruault, Y. (1990). Convergence of Adomian's method. Proceedings, $7^{\text {th }}$ International Conference on Mathematical and Computer Modeling. 14: 83-86.

Gray, J., Kassory, D.R., Tadjeran, H. and Zebib, A. (1982). Effect of significant viscosity variation on convective heat transport in water-saturated porous media, J. Fluid Mech., 117: 233-249.

Hossain, M.A. Khanafer, K., and Vafai, K. (2001). The effect of radiation on free convection flow of fluid with variable viscosity from a porous vertical plate. Int. J. Therm. Sci., 40:115-124. 
Ibanez, G., Cuevas, S. and Lopez de Haro, M. (2003).Minimization of entropy generation by asymmetric convective cooling. International Journal of Heat and Mass Transfer. 46:1321-1328.

Isreal-Cookey, C., Ogulu, A and Omubo-pepple , V.B. (2003) Influence of viscous dissipation on unsteady MHD free convection flow past an infinite heated vertical plate in a porous medium with time dependent suction. Int. journal of heat mass transfer. Vol.46. pp.2305-2311.

Jha, B.K., Yabo, I.B. and Lin, J. \{2017). Transient Natural Convection in an annulus with Thermal Radiation. Journal of Applied Mathematics, 8: 1351- 1366.

Kay, A. (2017).Comments on 'Combined effect of variable viscosity and thermal conductivity on free convection flow in a vertical channel using DTM' by J.C. Imavathi and M. Shekar. Meccanica, 52 (6): 1493-1494.

Lai, E.C. and Kulacki, E.A. (1990). The effect of variable viscosity on convective heat transfer along a vertical surface in a saturated porous medium. International Journal of Heat and Mass Transfer. 33(5):1028-1031.

Makinde, O.D. (2008). Entropy generation analysis for variable viscosity channel flow with non-uniform wall temperature. Journal of Applied Energy, 85:384-393.

Makinde, O.D., Olajuwon, B. I. and Gbolagade A.W. (2007). Adomian Decomposition Approach to a Boundary Layer Flow with Thermal Radiation past a Moving Vertical Porous Plate. International Journal of Applied Mathematics and Mechanics. 3(3):62-70.

Makinde, O.D., and Ogulu, A. (2011). The effect of thermal radiation on the heat and mass transfer flow of a variable viscosity fluid past a vertical porous plate permeated by a transverse magnetic field. Journal of Chemical Engineering Communications. 195:1575- 1584.

Magyari, E. and Pantokratoras, A. (2011). Note on the effect of thermal radiation in the linearized Rosssel and approximation on the heat transfer characteristics of various boundary layer flows. International Communications in Heat and Mass Transfer. 38: 554- 556.

Mehta, K.N. and Sood, S. (1992). Transient free convection flow with temperature-dependent viscosity in a fluid saturated porous medium, Int. J. Eng. Sci., 30:1083-1087.

Neild, D.A. and Bejan, A. (2013). Convection In porous media. $4^{\text {th }}$ edition. Springer, New York.
Ingham, D.B. and Pop, I. (1998) Transport phenomena in porous media. Pargamon. Oxford.

Robert, W. and Murray, R. S. (2010). Schaum's Oulines Series for Advanced Calculus. Third Edition.

Sahin,A.Z. (1999).Effect of variable viscosity on the entropy generation and pumping power in a laminar fluid flow through a duct subjected to constant heat flux. Journal of Heat and Mass Transfer. 35: 499-509.

Salem, A.M. (2007). Variable viscosity and thermal conductivity effects on MHD flow and heat transfer in viscoelastic fluid over a stretching sheet. Physics Letters A, 369(4): 315-322.

Salem, A.M. (2013). The Effects of Variable viscosity, Viscous Dissipation and Chemical Reaction on Heat and Mass Transfer Flow of MHD Micropolar Fluid alond a Permeable Stretching Sheet in a Non-Darcian Porous Medium.Hindawi Publishing Company. Mathematical Problem in Engineering. Volume 2013.

Seddeek, M.A. and Salama, E.A. (2007). The effects of temperature-dependent viscosity and thermal conductivity on unsteady MHD convective heat transfer past a semi-infinite vertical porous moving plate with variable suction, Computational Materials Science, $\quad$ 40(2):186-192.

Soundalgekar.V.M (1972) viscous dissipation effect on unsteady natural convective flow past an infinite vertical porous plate with constant suction. Int. journal of heat mass transfer.15:1253-1261.

Sparrow, E. M. and Cess. R. D. (1978). Radiation Heat Transfer, augmented edition. Hemisphere, Washington, D. C.

Yasutomi, S., Bair, S. and Winer, W.O. (1984). An application of a free volume model to lubricant rheology I. Dependence on viscosity on temperature and pressure, J. Tribol. Trans. ASME., 106:291-303.

Yabo, I.B., Jha, B.K. and Lin, J. (2016). Combined Effects of Thermal Diffusion and Diffusion-Thermo Effects on Transient MHD Natural Convection and Mass Transfer Flow in a Vertical Channel with Thermal Radiation. Journal of Applied Mathematics. 6: 2354-2373.

Yusuf, A.B. and Ajibade, O.A. (2018a). Unsteady Natural Convection Flow through a vertical Porous Channel filled with Porous Materials under the effect of Thermal Radiation. FUDMA Journal of Sciences, 2(2):101-115. 
Yusuf, A.B. and Ajibade, O.A. (2018b). Combined Effects of Variable Viscosity and Thermal Radiation on Natural Convection Flow through a vertical Porous Channel, FUDMA Journal of Sciences, 2(2):273-287.

Yusuf, A.B. and Ajibade, O.A. (2019). Variable Fluid Viscosity and Thermal Radiation Effects on Natural Convection Couette Flow through a vertical Porous Channel. Journal of Advances in Mathematics and Computer Science, 13(1):1-17. use, distribution, and reproduction in any medium, provided the original work is cited appropriately. 